\title{
BMJ Open Dynamics and Determinants of HPV Infection: The Michigan HPV and Oropharyngeal Cancer (M-HOC) Study
}

Marisa C Eisenberg, ${ }^{1}$ Lora P Campredon, ${ }^{1}$ Andrew F Brouwer, ${ }^{1}$ Heather M Walline, ${ }^{2}$ Brittany M Marinelli, ${ }^{2}$ Yan Kwan Lau, ${ }^{1}$ Trey B Thomas, ${ }^{2}$ Rachel L Delinger, ${ }^{1}$ Taylor S Sullivan, ${ }^{1}$ Monica L Yost, ${ }^{1}$ Christine M Goudsmit, ${ }^{2}$ Thomas E Carey, ${ }^{2}$ Rafael Meza ${ }^{1}$

To cite: Eisenberg MC, Campredon LP, Brouwer AF, et al. Dynamics and Determinants of HPV Infection: The Michigan HPV and Oropharyngeal Cancer (M-HOC) Study. BMJ Open 2018;8:e21618. doi:10.1136/ bmjopen-2018-021618

- Prepublication history for this paper is available online. To view these files, please visit the journal online (http://dx.doi. org/10.1136/ bmjopen-2018-021618)

Received 15 February 2018 Revised 1 August 2018 Accepted 18 August 2018

\section{Check for updates}

(C) Author(s) (or their employer(s)) 2018. Re-use permitted under CC BY-NC. No commercial re-use. See rights and permissions. Published by BMJ.

${ }^{1}$ Department of Epidemiology, University of Michigan, Ann Arbor, Ann Arbor, Michigan, USA 'Department of Otolaryngology, University of Michigan, Ann Arbor, Ann Arbor, Michigan, USA

Correspondence to Dr Marisa C Eisenberg; marisae@umich.edu and Dr Rafael Meza; rmeza@umich.edu

\section{ABSTRACT}

Introduction Human papillomavirus (HPV) is the primary cause of cervical and other anogenital cancers and is also associated with head and neck cancers. Incidence of HPV-related oropharyngeal squamous cell cancers (OPSCCs) is increasing, and HPV-related OPSCCs have surpassed cervical cancer as the most common HPVrelated cancer in the USA. Given the multisite nature of $\mathrm{HPV}$, there is strong interest in collecting data from both genital and oral sites, as well as associated data on social and sexual behaviours. The overarching goal of this study is to evaluate patterns of oral HPV infection incidence, clearance and persistence and their relationship to sexual behaviour history.

Methods and analysis Participants are recruited from two populations: college students at a large public university and general population from the surrounding area. At the first study visit, participants complete a detailed sexual history, health and behaviour questionnaire. Follow-up visits occur every 3-4 months over 3 years, when participants complete an abbreviated questionnaire. All participants provide a saliva sample at each visit, and eligible participants may provide a cervicovaginal selfswab. Genetic material isolated from specimens is tested for 15 high-risk and 3 low-risk HPV types. Statistical analyses will examine outcome variables including HPV prevalence, incidence, persistence and clearance. Logistic regression models will be used to estimate odds ratios and $95 \%$ confidence intervals for associations between the outcomes of interest and demographic/behavioural variables collected in the questionnaires. The longitudinal HPV infection data and detailed sexual history data collected in the questionnaires will allow us to develop individual-based network models of HPV transmission and will be used to parameterise multiscale models of HPVrelated OPSC carcinogenesis.

Ethics and dissemination This study has been approved by the University of Michigan Institutional Review Board. All participants are consented in person by trained study staff. Study results will be disseminated through peer-reviewed publications.

\section{INTRODUCTION}

Infectious disease agents account for approximately $20 \%$ of cancers worldwide. ${ }^{1}$ The

\section{Strengths and limitations of this study}

- Longitudinal design with 3-4 study visits per participant per year allows for analysis of incidence, persistence and clearance of oral human papillomavirus (HPV).

- Collection of both oral and cervicovaginal samples allows for comparison of infections across sites.

- Low prevalence of high-risk oral HPV may limit our ability to connect HPV infection dynamics to demographic, sexual and behavioural variables.

- Participant-dependent saliva production and swishing effectiveness may result in variable sample quality, but this concern is mitigated by PCR sensitivity.

- Our HPV test separately identifies 15 high-risk genotypes but only 3 low-risk genotypes and thus may not provide a complete picture of the patterns of mucosal HPV types.

human papillomavirus (HPV) is the primary cause of cervical and most anogenital cancers, as well as an increasing fraction of head and neck (HN) cancers. ${ }^{23}$ Indeed, incidence of HPV-related oropharyngeal squamous cell cancers (OPSCCs) is increasing, ${ }^{4-7}$ and HPV-related OPSCCs have surpassed cervical cancer as the most common HPV-related cancer in the USA. ${ }^{3}$ At the University of Michigan (UM) Cancer Center, over $80 \%$ of patients with OPSCC have high-risk HPV detected in their cancers. ${ }^{8}$ Although many HPV infections are benign, high-risk HPV infections can disrupt several key cancer regulation pathways, including those involving tumour suppressors p53 and pRb. ${ }^{9}{ }^{10}$

The underlying reasons behind the significant rise in HPV-related OPSCG rates in the USA are unclear. From a prevention perspective, addressing HPV-related OPSCC begins with an understanding of the epidemiology of the virus, both in the oral cavity and more broadly. Estimates from the National Health and Nutrition Examination Survey 
(NHANES) suggest that oral HPV prevalence in the USA is about $11 \%-12 \%$ in men and $3 \%-4 \%$ in women. ${ }^{11-14}$ However, while oral HPV infections may clear relatively quickly (the HPV in Men (HIM) trial estimated a mean clearance time of approximately 7 months ${ }^{15}$ ), persistent infections may lead to cancer after several decades. (Although there are no precise estimates of time to cancer for OPSCCs, cervical lesions are estimated to progress to cancer in $10-30$ years $^{16}$ ).

While studies are increasingly treating HPV as a multisite disease, the mechanisms underlying the relationship between oral, genital and anal HPV infections are not understood, although autoinoculation is increasingly implicated. ${ }^{17} 18$ The HPV vaccine further complicates the epidemiological picture: HPV vaccination is expected to have an impact on the rates of oral HPV incidence, at least for those genotypes included in the vaccine. $^{12}{ }^{19}$ Countries with more aggressive vaccination campaigns, such as Australia, have already seen a reduction in HPV-related genital warts. ${ }^{20}{ }^{21}$ It remains to be seen whether HPV vaccination will ultimately reduce the incidence and mortality of HPV-related HN cancers.

Many open questions remain regarding oral HPV transmission, epidemiology, infection and persistence, the mechanisms of HPV-related HN carcinogenesis, and the connection between the ongoing oral HPV epidemic and the rising OPSCC incidence. The knowledge gaps surrounding the mechanisms and epidemiology of HPV in the oral cavity hamper our ability to develop more effective measures to prevent oral HPV infections and, in turn, reduce the burden of $\mathrm{HN}$ cancers. Prevalence studies can only draw associations between the burden and probable risk factors. Thus, longitudinal studies with frequent testing and assessment of recent sexual behaviour are needed to understand HPV transmission, persistence and clearance and to associate these events with certain behaviours. Here, we describe the methods, study design and initial study population characteristics of a longitudinal study of oral HPV incidence, clearance and related sexual behaviours; this study and its cervicovaginal HPV substudy are the epidemiological arms of the Michigan
HPV and Oropharyngeal Cancer (MHOC) Study. Our research will contribute to a better understanding of the complex factors affecting patterns of HPV infection. This study will inform predictive and mechanistic models and develop effective prevention strategies for OPSCC and other HPV-related cancers.

\section{METHODS AND ANALYSIS \\ Objective}

The overarching goal of this study is to evaluate patterns of oral HPV infection incidence and clearance and their relationship to sexual history and sexual behaviours. Participants are recruited to the study from two populations: college students at UM (a large public university) and the general population of the surrounding area. As a secondary goal, we will also compare HPV infection patterns in the oral and cervicovaginal sites as part of a substudy examining cervicovaginal HPV infection.

\section{Subject enrolment and consent}

Here, we describe the process for subject enrolment and consent (diagrammed in figure 1).

\section{Identification of study subjects}

Participants are continuously recruited by one of three methods. (1) Advertisement: we place recruitment announcements in UM health clinics and area community sites (eg, libraries, coffee shops, bus stops), as well as on UM clinical research volunteer's website (www. umclinicalstudies.org). In this method, individuals who are potentially interested in participating in the study contact the study coordinator to determine eligibility. (2) Screening clinics: we approach participants of the UM health system free throat cancer screening clinic to invite eligible participants to join the study. (3) Student residence halls: once per year, we set up information tables in or near UM residence halls and invite eligible students to join the student.

Eligibility criteria. Volunteers are considered for enrolment if they

Figure 1 Flow diagram for the study detailing recruitment methods, visit structure (baseline and follow-ups) and the cervicovaginal substudy. H\&N, head and neck. 
- Are age 18 or older.

- Do not have a history of HN cancer.

- Are willing to return for a follow-up every 3-4 months over 3 years.

- Are able and willing to provide written informed consent.

To participate in the optional cervicovaginal substudy, participants must additionally

- Have a vagina.

- Not be pregnant.

- Not be menstruating at the time of the study visit.

\section{Consent}

We document informed consent for all willing and eligible participants before enrolment in the study. Separate, additional consent is obtained prior to enrolment in the cervicovaginal substudy. All study subjects are given the opportunity to consent to storage of residual specimens collected in this study for future research purposes. Specimens collected from subjects who do not consent to specimen banking will be destroyed after completion of the analyses of this study.

\section{Benefit to participants}

Since we are not using a clinical test, our Institutional Review Board approval does not allow returning individual HPV test results to participants, but population-level results will be disseminated through newsletters after peer-reviewed publication. Staff are knowledgeable and available to discuss vaccination and screening with interested participants. Because of the nature of the sexual and behavioural questionnaire, we also have pamphlets for the UM Sexual Assault Prevention and Awareness Center available to participants.

\section{Enrolment and statistical power}

Phase I of this study recruited between April 2015 and December 2017, enrolling 395 participants. At this sample size and level of significance 0.05 , we will detect the difference at baseline between two equally sized populations with HPV prevalence $10 \%$ and $20 \%$ with $80 \%$ power. Assuming each participant completes 10 visits, we will detect the difference between $10 \%$ and $13 \%$ ever HPV positive with more than $80 \%$ power. At the time of submission, 321 participants had completed at least one follow-up visit, and 1693 baseline and follow-up visits had been completed. Follow-up visits are ongoing. Pending funding, phase II is anticipated to recruit a similar number of participants (potentially with additional study locations as well).

\section{Data collection}

We administer surveys and collect biological specimens as described below.

\section{Social and sexual behaviour survey}

All study participants complete a comprehensive questionnaire at the baseline visit and an abbreviated questionnaire at all subsequent visits. The questionnaire
Table 1 Categories and numbers of questions asked in Michigan HPV and Oropharyngeal Cancer (MHOC) study baseline and follow-up questionnaire.

\begin{tabular}{lcc}
\hline & \multicolumn{2}{c}{ Number of questions } \\
\cline { 2 - 3 } Question category & Baseline & Follow-up \\
\hline Demographic & 8 & 5 \\
Oral health and cancer & 5 & 5 \\
STI testing and diagnosis & 17 & 12 \\
Sexual health and vaccinations & 25 & 16 \\
Sexual behaviour & 70 & 6 \\
Sexual partners & 22 & 18 \\
Substance use & 20 & 16 \\
\hline
\end{tabular}

STI, sexually transmitted infection.

consists of questions related to demographic information; behaviour, including alcohol, cigarette, cigar, tobacco, and other drug use; and sexual practices, sexual history, sexual healthcare (including HPV vaccination status), general health and history of sexually transmitted infections (STIs). The baseline questionnaire collects a complete sexual history, with subsequent follow-up visits providing more recent information and updates. The questions regarding sexual behaviours and history are designed to provide detailed information about the timing of different sexual partnerships and sexual practices in each sexual partnership and form a longitudinal, egocentric sexual network data set. Table 1 details the categories and numbers of questions given in both the baseline and follow-up surveys. The questionnaire is administered electronically via Qualtrics in a quiet, private room. Participants are asked to enter their unique study identification number at the beginning of the questionnaire. Participants are free to skip or otherwise not answer any question.

\section{Oral specimen collection}

Two millilitres of saliva and oral rinse specimen are collected at each visit (ie, baseline and every 3-4 months for 3 years) using Oragene RE-100 kits. ${ }^{22}$ Participants may use a sweetener or candy to stimulate saliva production if needed.

In the initial phase of the study, we tested several alternative saliva collection methods: Scope-brand mouthwash (administered as in NHANES ${ }^{23}$ ), and two commercially available kits developed for detection of DNA and RNA in saliva, the OM-505 (suitable for DNA, stable for weeks at room temperature) and RE-100 (designed to protect RNA, stable for 60 days at room temperature). ${ }^{22}{ }^{24} \mathrm{We}$ were able to detect DNA from saliva in all three methods but were only able to reliably recover RNA from the RE-100 kit sample. We selected the RE-100 kits for use in the study so that we could isolate both DNA and RNA from a single sample. 


\section{Cervicovaginal swab collection}

Participants fulfilling the eligibility criteria are given the option at each study visit to provide a self-collected cervicovaginal swab sample using a HerSwab ${ }^{25}$ in addition to the oral specimen. The HerSwab is a vaginal swab designed to sample near the cervix. Participants self-administer the swab by inserting the tip of the HerSwab into the vagina; turning the crank so that the brush is fully extended; removing the swab; turning the crank the opposite direction to retract the brush back into the tip; and placing the HerSwab into a provided resealable plastic bag. The participants are provided with the printed, graphical instructions designed by the manufacturer and collect their sample in a restroom. Staff facilitating the study are available to review the procedure and answer any participant questions.

\section{Cervicovaginal swab acceptability survey}

All study participants who provide a cervicovaginal sample complete a questionnaire after each cervicovaginal self-collection that asks about comfort, ease of use and subjective assessment of the self-collection procedure. $^{26}$ The questionnaire is administered electronically via Qualtrics.

\section{Follow-up visit schedule}

Participants are followed for up to 3 years after their baseline visit, for a total of up to 12 study visits. The study prioritises longitudinal follow-up over regular follow-up but strives to achieve both. Therefore, if participants miss a follow-up visit, they are not excluded and instead continue the study when next available. This protocol facilitates research in the student population, as students are often gone for extended periods of time on scheduled breaks.

\section{Sample processing and analysis}

Sample processing and DNA extraction differs for oral and cervicovaginal samples, but DNA analysis is the same for both kinds of samples.

\section{Oral sample processing}

DNA isolation from saliva samples is performed by first splitting the sample in half, half for DNA and half for RNA. The saliva is heated to $50^{\circ} \mathrm{C}$ for $60 \mathrm{~min}$, and $1 / 25 \mathrm{th}$ volume of prepIT-L2P (DNA Genotek) is added. Sample is incubated for $10 \mathrm{~min}$ at $4^{\circ} \mathrm{C}$ and spun for $20 \mathrm{~min}$ at 4100 revolutions per minute. After that the supernatant is added to equal volumes of $95 \%$ ethanol to precipitate the DNA. The DNA is pelleted, washed with $200 \mu \mathrm{L}$ of $70 \%$ ethanol and dried. The DNA pellet is rehydrated with Tris-EDTA buffer and quantified with picogreen (Invitrogen P11496) or the QuBit (Invitrogen Q32850). RNA is isolated from samples that have tested positive for HPV DNA. RNA isolation from saliva samples is performed using the Oragene RNA isolation method according to the manufacturer's instruction which includes QIAgen's RNeasy Micro Kit (74004). In short, the samples are heated to $50^{\circ} \mathrm{C}$ for $60 \mathrm{~min}, 95^{\circ} \mathrm{C}$ for $15 \mathrm{~min}$, and $20 \mu \mathrm{L}$ neutralisation solution is added. Samples then are incubated on ice for $10 \mathrm{~min}$ followed by the RNeasy Kit protocol. The RNA is then converted to cDNA for further testing. The cDNA synthesis is preformed using Invitrogen's Superscript III protocol and reagents (18080-044).

\section{Cervicovaginal sample processing}

After collection, the HerSwab is soaked for $1 \mathrm{~min}$ in $20 \mathrm{~mL}$ of ThinPrep solution and subsequently swished vigorously for $1 \mathrm{~min}$. The $20 \mathrm{~mL}$ solution is then split evenly into two $15 \mathrm{~mL}$ nuclease-free conical tubes-one for RNA processing and one for DNA-and the tube for RNA is centrifuged. The pellet for RNA use is resuspended in RNAlater for isolation and later study. DNA and RNA processing is the same as above. For RNA isolation, the cells in RNAlater are pelleted by centrifugation and RNA is isolated using the RNeasy as described above. Qiagen reagents are used for DNA isolation. The cells in the ThinPrep sample are pelleted, resuspended in cell lysis buffer, incubated with Proteinase $\mathrm{k}$, treated with protein precipitation buffer, vortexed vigorously, pelleted and placed on ice for $10 \mathrm{~min}$. The supernatant is transferred to isopropanol in glycogen solution mixed, and the DNA pellet is washed twice with cold ethanol, rehydrated in hydration solution and dissolved at $65^{\circ} \mathrm{C}$, and DNA is quantified with picogreen or QuBit.

\section{DNA analysis}

DNA and cDNA samples are assayed using a previously described, highly sensitive method. ${ }^{8}$ Briefly, multiplex competitive PCR amplification of the heterogeneous E6 region of 15 discrete high-risk (16, 18, 31, 33, 35, 39, $45,51,52,56,58,59,66,68,73)$ and 3 low-risk $(6,11$, 90) HPV types is performed, followed by probe-specific single-base extension. Extension products are loaded onto a matrix silicon chip array and separated by size using matrix-assisted laser desorption/ionisation time-offlight mass spectroscopy, allowing detection of any HPV types present in the sample.

\section{STATISTICAL ANALYSIS}

Outcomes of interest include, but are not limited to, HPV prevalence (detection of HPV, or detection of a specific HPV genotype), incidence (detection of HPV in a previously uninfected person, or detection of a specific HPV genotype in a person who previously tested negative for that genotype), persistence (detection of HPV at subsequent study visits, or detection of specific genotypes at subsequent study visits), and clearance (non-detection of HPV in a previously infected person, or non-detection of a specific HPV genotype in a person previously infected by that genotype). Other patterns of HPV detection, such as patterns of intermittent detection of the same genotype or detection of an HPV genotype at the oral site after previous detection at the genital site (or vice versa), will be considered. We will adjust for the time between visits as 
appropriate. Presence of RNA in addition to DNA will be used to distinguish between active and latent infections.

Covariates of interest include, but are not limited to, sex, age, student status, HIV status, sexual orientation, HPV vaccine status, circumcision status, number of vaginal, oral, and anal sexual partners, timing and nature of recent sexual activity, use of sexual protection and substance use. Demographic and sexual behaviour differences among subpopulations will be considered.

Logistic regression models will be used to estimate odds ratios and $95 \%$ confidence intervals for associations between the outcomes of interest and the demographic/ behavioural variables. Variables that are significant in univariate analysis or are considered to be relevant based on a priori knowledge will be evaluated in multiple logistic regression models. Cox regression models will be used to investigate the effect of covariates on clearance. Analyses will be performed in SAS or R statistical software.

\section{MODELLING ANALYSIS}

The longitudinal HPV infection data and detailed sexual partner and sexual history data collected in the questionnaires will allow us to develop individual-based network models of HPV transmission. While the data from the study are necessarily egocentric (ie, we cannot link partners reported by participants to any specific individual in or out of the study to form a full sexual network), they can nonetheless be used to directly parameterise simulation models of transmission. The models we develop can match the timing of partner switching, numbers of partners and type of sexual contact, as well as a range of behavioural and demographic covariates as well as HPV infections and clearance times. We will draw from the measured distributions and correlation patterns of these variables in our population to generate simulated populations with sexual behaviour patterns similar to those measured in our study (eg, using approaches based on configuration model methods ${ }^{27}$ to connect our simulated sexual network based on partner history data). This will allow us to generalise our statistical model findings to different populations and to examine counterfactual scenarios of alternative HPV vaccine coverage levels. Similar models have been developed for other studies, both for STIs and other infectious diseases. ${ }^{28-33}$ This study is designed to specifically inform such modelling analyses.

\section{Patient and public involvement}

Patients and the public were not involved in the development of this study. Study results will be disseminated to the public through peer-reviewed publications as described in the ethics and dissemination section. Additionally, the newsletter described will be made accessible to the public through the study website.

\section{Ethics and dissemination}

This study was deemed to not require additional safety monitoring beyond the monitoring of the institutional review board. All study staff interacting with participants and participant data complete the University of Michigan Program for Education and Evaluation in Responsible Research and Scholarship (PEERRS) prior to beginning the project. ${ }^{31}$ Study results will be disseminated through a series of peer-reviewed publications. Study participants will receive a study results newsletter written for a general audience.

\section{DISCUSSION}

The knowledge gained from this study will contribute to the field of HPV research and will be disseminated via peer-reviewed publications and presentations at national and international conferences. This study will provide improved estimates of HPV clearance rates in the oral cavity, due to the short follow-up times, and will also allow us to examine how HPV clearance is affected by a range of factors, including coinfection with multiple HPV types. More broadly, this study will improve our understanding of the relationships between behaviour patterns and HPV, helping to elucidate how sexual behaviours, numbers of partners and other behaviours (eg, alcohol and substance use) relate to HPV infection patterns. Additionally, this study will provide detailed data on HPV transmission in the college student population-a key population who are also seeing changing vaccination patterns as HPV vaccine coverage improves. This will allow us to further examine the impact of HPV vaccination on the incidence and prevalence of a range of vaccine types (both those covered by the vaccine and others). The study questionnaire and longitudinal design also are also useful in developing sexual network models of transmission, which will allow us to examine alternative vaccination strategies and generalise to other populations.

\section{Limitations and strengths}

Because oral HPV prevalence in the USA is relatively low (prevalence of high-risk oral HPV among adults 18-69 was $4.0 \%$ in 2011-2014: $6.8 \%$ in men and $1.2 \%$ in women ${ }^{14}$ ), there is a risk that the number of HPV-positive samples in the study will be low, limiting our abilities to draw inference between HPV infection and the demographic and behavioural variables of the participant. The quality of our saliva and oral rinse specimens may depend on the saliva production and swishing efficacy of each participant, although this is mitigated by the sensitivity of the PCR analysis. Finally, we only test for 18 genotypes, which, although we cover all high-risk types, may not give as complete a picture of patterns of mucosal HPV infection.

Instead of identifying risk factors through associations in cross-sectional prevalence analyses, we will use a longitudinal approach with frequent oral and cervicovaginal HPV testing and updated detailed sexual behaviour questionnaires. This will allow for understanding prevalence of HPV and incidence, persistence and clearance. Some studies have also adopted a longitudinal approach $\left(\mathrm{eg},{ }^{34-38}\right)$, but many have had long times between follow-up 
or have focused only on sex or only on genital infection sites. In the MHOC study, we use short follow-up times, with 3-4 visits per participant per year. We focus primarily on oral infection in both men and women but also collect cervicovaginal samples to assess multisite infections.

Acknowledgements We would like to thank Professors Carol Bradford, Paul Hoff, Norm Hogikyan, Trachette Jackson, James Koopman, Kelly Malloy, John McHugh, Erin McKean, Mark Prince, Jamie Riddell, Laura Rozek, Andrew Shuman, Matthew Spector, Jeffrey Stanley, Jeremy Taylor, Francis Worden, and Gregory Wolf, for their helpful comments, advice, and support. We also thank our collaborators, study and laboratory staff, and students: Miranda West, Thankum Nair, Lisa Petersen, Sheila Terrones, Chad Brenner, Emily Bellile, Justin Bell, Tori Melandez, and Eliyas Asfaw, Christian Alvarez, Kristin Bevilacqua, Pian Pian Cao, Alanna Clark, Jesse Contreras, Rachel Delinger, Greg Foakes, Anna Gottschlich, Manila Hada, Christina Hanson, Nadine Jawad, Bala Naveen Kakaraparthi, Alexandra Kalabat, Jung Woo Lee, Jodi McCloskey, Ivan Montoya, Anna Morris, Chinmay Pandit, Derrick Sund, Kayla Stanek, Peter Tortora, Taylor Vandenberg, and Lucy Yang. We would like to thank all of our staff and our study participants, without whom this study would not have been possible.

Contributors The study was conceptualised and funding was obtained by MCE, TEC and RM. Methodology and original protocols were developed by MCE, LPC, AFB, HMW, BMM, YKL, TBT, RLD, CMG, TEC and RM. Project administration is managed by LPC and YKL. MCE, LPC, AFB, HMW, BMM, YKL, CMG, TEC and RM are responsible for the supervision of study implementation, staff and students. The original draft was prepared by MCE with the assistance of LPC, TSS and MLY. Review and editing were completed by MCE, LPC, AFB, HMW, BMM, YKL, TEC and RM.

Funding This work was supported by the National Institutes for Health Grant U01CA182915, the University of Michigan MCubed Program, a pilot grant from the University of Michigan Comprehensive Cancer Center's Cancer Epidemiology \& Prevention Program (supported by the National Cancer Institute of the National Institutes of Health grant P30CA046592), and the Michigan Institute for Clinical and Health Research (NIH Grant UL1TR000433).

Competing interests None declared.

Patient consent Not required.

Ethics approval The Michigan HPV and Oropharyngeal Cancer study was approved by the University of Michigan Institutional Review Board on 26 August 2014 (IRB \# HUM00090326).

Provenance and peer review Not commissioned; externally peer reviewed.

Open access This is an open access article distributed in accordance with the Creative Commons Attribution Non Commercial (CC BY-NC 4.0) license, which permits others to distribute, remix, adapt, build upon this work non-commercially, and license their derivative works on different terms, provided the original work is properly cited, appropriate credit is given, any changes made indicated, and the use is non-commercial. See: http://creativecommons.org/licenses/by-nc/4.0/.

\section{REFERENCES}

1. Bouvard V, Baan R, Straif K, et al. A review of human carcinogensPart B: biological agents. Lancet Oncol 2009;10:321-2.

2. Gillison ML, Alemany L, Snijders PJ, et al. Human papillomavirus and diseases of the upper airway: head and neck cancer and respiratory papillomatosis. Vaccine 2012;30 Suppl 5(Suppl 5):F34-F54.

3. Jemal A, Simard EP, Dorell C, et al. Annual Report to the Nation on the Status of Cancer, 1975-2009, featuring the burden and trends in human papillomavirus(HPV)-associated cancers and HPV vaccination coverage levels. J Natl Cancer Inst 2013;105:175-201.

4. Chaturvedi AK, Engels EA, Anderson WF, et al. Incidence trends for human papillomavirus-related and -unrelated oral squamous cell carcinomas in the United States. J Clin Oncol 2008;26:612-9.

5. Chaturvedi AK, Engels EA, Pfeiffer RM, et al. Human papillomavirus and rising oropharyngeal cancer incidence in the United States. $J$ Clin Oncol 2011;29:4294-301.

6. Chaturvedi AK, Anderson WF, Lortet-Tieulent J, et al. Worldwide trends in incidence rates for oral cavity and oropharyngeal cancers. $J$ Clin Oncol 2013;31:4550-9.

7. Chaturvedi AK, D'Souza G, Gillison ML, et al. Burden of HPV-positive oropharynx cancers among ever and never smokers in the U.S. population. Oral Oncol 2016;60:61-7.
8. Walline HM, Komarck C, McHugh JB, et al. High-risk human papillomavirus detection in oropharyngeal, nasopharyngeal, and oral cavity cancers: comparison of multiple methods. JAMA Otolaryngol Head Neck Surg 2013;139:1320.

9. Doorbar J, Quint W, Banks L, et al. The biology and life-cycle of human papillomaviruses. Vaccine 2012;30 Suppl 5(Suppl 5):F55-F70.

10. McLaughlin-Drubin ME, Münger K. Oncogenic activities of human papillomaviruses. Virus Res 2009;143:195-208.

11. Gillison ML, Broutian T, Pickard RK, et al. Prevalence of oral HPV infection in the United States, 2009-2010. JAMA 2012;307:693-703.

12. Brouwer AF, Eisenberg MC, Carey TE, et al. Trends in HPV cervical and seroprevalence and associations between oral and genital infection and serum antibodies in NHANES 2003-2012. BMC Infect Dis 2015;15:575.

13. Sonawane K, Suk R, Chiao EY, et al. Oral Human Papillomavirus Infection: Differences in Prevalence Between Sexes and Concordance With Genital Human Papillomavirus Infection, NHANES 2011 to 2014. Ann Intern Med 2017;167:714-24.

14. McQuillan G, Kruszon-moran D, Markowitz LE, et al. Prevalence of HPV in Adults Aged 18-69: United States, 2011-2014. NCHS Data Brief 2017:280.

15. Kreimer AR, Pierce Campbell CM, Lin HY, et al. Incidence and clearance of oral human papillomavirus infection in men: the HIM cohort study. Lancet 2013;382:877-87.

16. McCredie MR, Sharples KJ, Paul C, et al. Natural history of cervical neoplasia and risk of invasive cancer in women with cervical intraepithelial neoplasia 3: a retrospective cohort study. Lancet Oncol 2008:9:425-34

17. Pamnani SJ, Nyitray AG, Abrahamsen M, et al. Sequential Acquisition of Anal Human Papillomavirus (HPV) Infection Following Genital Infection Among Men Who Have Sex With Women: The HPV Infection in Men (HIM) Study. J Infect Dis 2016;214:1180-7.

18. Ranjeva $\mathrm{S}$, Baskerville $\mathrm{EB}$, Dukic $\mathrm{V}$, et al. Recurring infection with ecologically distinct human papillomavirus (HPV) types explains high prevalence and diversity. bioRxiv, 2017.

19. Markowitz LE, Liu G, Hariri S, et al. Prevalence of HPV After Introduction of the Vaccination Program in the United States. Pediatrics 2016;137:e20151968-9.

20. Ali H, Donovan B, Wand H, et al. Genital warts in young Australians five years into national human papillomavirus vaccination programme: national surveillance data. BMJ 2013;346:f2032-2032.

21. Chow EP, Read TR, Wigan R, et al. Ongoing decline in genital warts among young heterosexuals 7 years after the Australian human papillomavirus (HPV) vaccination programme. Sex Transm Infect 2015;91:214-9.

22. DNA Genotek, Inc, 2017. Oragene-RNA (RE-100). http://www. dnagenotek.com/US/products/RE100.html

23. Centers for Disease Control and Prevention, 2009. National Health and Nutrition Examination Survey (NHANES): HPV rinse. https:// wwwn.cdc.gov/nchs/data/nhanes/2009-2010/manuals/HPV.pdf

24. DNA Genotek, Inc, 2017. OMNIgene-ORAL (OM-505). http://www. dnagenotek.com/US/products/OM505

25. Eve Medical. Eve Medical products: HerSwab. 2017. http://www.evemedical.com/products

26. Gottschlich A, Rivera-Andrade A, Grajeda E, et al. Acceptability of Human Papillomavirus Self-Sampling for Cervical Cancer Screening in an Indigenous Community in Guatemala. J Glob Oncol 2017;3:444-54.

27. Newman M. Networks: An Introduction: Oxford University Press, 2010.

28. Petrie JG, Eisenberg MC, Ng S, et al. Application of an IndividualBased Transmission Hazard Model for Estimation of Influenza Vaccine Effectiveness in a Household Cohort. Am J Epidemiol 2017;186:1380-8.

29. Aiello AE, Simanek AM, Eisenberg MC, et al. Design and methods of a social network isolation study for reducing respiratory infection transmission: The eX-FLU cluster randomized trial. Epidemics 2016;15:38-55.

30. Fan K, Eisenberg M, Walsh A, et al. Hierarchical graph-coupled $\mathrm{hmms}$ for heterogeneous personalized health data: In. Proceedings of the 21th ACM SIGKDD International Conference on Knowledge Discovery and Data Mining: ACM, 2015:239-48.

31. Kaplan EH, Heimer R. HIV prevalence among intravenous drug users: model-based estimates from New Haven's legal needle exchange. $J$ Acquir Immune Defic Syndr 1992;5:163-9.

32. Sloot PMA, Ivanov SV, Boukhanovsky AV, et al. Stochastic simulation of HIV population dynamics through complex network modelling. Int $J$ Comput Math 2008;85:1175-87.

33. Cohen T, Colijn C, Finklea B, et al. Exogenous re-infection and the dynamics of tuberculosis epidemics: local effects in a network model of transmission. J R Soc Interface 2007;4:523-31. 
34. University of Michigan. Responsible Conduct of Research (RCR) Training, 2017. http://research-compliance. umich.edu/responsibleconduct-research-rcr-training.

35. Ho GY, Bierman R, Beardsley L, et al. Natural history of cervicovaginal papillomavirus infection in young women. $N$ Engl $J$ Med 1998;338:423-8.

36. Franco EL, Villa LL, Sobrinho JP, et al. Epidemiology of acquisition and clearance of cervical human papillomavirus infection in women from a high-risk area for cervical cancer. J Infect Dis 1999;180:1415-23.
37. Molano M, Van den Brule A, Plummer M, et al. Determinants of clearance of human papillomavirus infections in Colombian women with normal cytology: a population-based, 5-year follow-up study. Am J Epidemiol 2003;158:486-94.

38. Goodman MT, Shvetsov YB, McDuffie K, et al. Sequential acquisition of human papillomavirus (HPV) infection of the anus and cervix: the Hawaii HPV Cohort Study. J Infect Dis 2010;201:1331-9. 